\title{
Dietary Trans Fatty Acids: Impact on Serum Lipoproteins and Coronary Heart Disease
}

\author{
Raghib Ahsan*
}

\begin{abstract}
Recently, there has been considerable concern on the atherogenic potential of Trans Fatty Acids (TFA) present in the food products produced with hydrogenated vegetable and marine oils. Health authorities and Food regulatory agencies across the world, including WHO and FAO, have suggested food manufacturers to lower TFA content in their food products and called for a mandatory food labeling on packs for consumer information and protection.
\end{abstract}

TAJ 2005; 18(2): 144-148

\section{Introduction}

Occlusive arterial disease of the heart, called Coronary Heart Disease (CHD) is very common in the developed countries and is the single most common cause of premature death. Its incidence is increasing rapidly and in an alarming fashion in the underdeveloped and developing countries also.

CHD has a multifactorial etiology, among which dyslipidemias are well explained. Dietary lipid, frequently resulting from faulty dietary habits, plays a very important role in causing dyslipidemias and consequently CHD. Role of saturated animal fat and cholesterol in inviting an unfavorable lipoprotein profile and that of unsaturated vegetable oil, a favorable lipoprotein profile is well accepted. Recently, there has been considerable concern on the atherogenic potential of Trans Fatty Acids (TFA) present in food products produced with hydrogenated vegetable and marine oils. The negative impact of TFAs on cardiovascular system has been highlighted only during the last two decades. In 1990s, several studies around the world concluded that hydrogenated fats containing TFAs are harmful for heart. Health authorities and Food regulatory agencies across the world, including WHO and FAO, have suggested food manufacturers to lower TFA content in their food products and called for a mandatory food labeling on packs for consumer information and protection.

\section{Trans Fatty Acids}

Unsaturated fatty acids exhibit geometric isomerism on the orientation of atoms or groups around it's double bond axis. ${ }^{3}$ Atoms or acyl groups, present on the same side of the double bond forms a cis-configuration, whereas those present on the opposite side of the double bond forms a trans-configuration. ${ }^{3}$ Oleic acid is a cis isomer while Elaidic acid is a trans isomer (Fig 1).

Trans fatty acids are a group of "deviant" fatty acids with the bulk of it's members occurring as the $\triangle^{9-11}$ isomers of Elaidic acid. Dietary TFAs come from two main sources. Firstly, through fermentation by bacteria in the gut of ruminants with TFA appearing in the milk and dairy products (milk, butter, cheese etc.). Secondly, during commercial hardening, by partial hydrogenation of edible liquid (vegetable) oils, for enhancement of product shelf-life (stabilizes the oil and prevents 
rancidity) or for use by the food industries in solid-fat applications such as in margarines, vegetable ghee, Vanaspati (dalda), shortenings, confectionary fats etc., and as frying media for fast foods items.

\section{TFAs, Atherosclerosis and CHD}

Occlusion of coronary arteries usually occurs due to atherosclerosis and it's complications. Though some basic characteristics of atherosclerosis are still poorly understood, it is well accepted that some generalized risk factors like, smoking, obesity, blood lipid disorders, hypertension, faulty dietary habits, insulin resistance, dietary deficiency of antioxidants, physical inactivity, lifestyle etc. ${ }^{1,2}$ predispose to it's development. However, among these risk factors abnormalities of plasma lipoprotein and derangements in lipid metabolism are more firmly established and best understood risk factors for atherosclerosis. Thus, hypercholesterolemia and other abnormalities in lipid metabolism contribute a major risk factor in atherosclerosis as well as CHD.

Almost all lipoprotein disorders or dyslipidemias (hyperlipidemia) result from a complex interaction between genetic predisposition and dietary indiscretion. ${ }^{2}$ Diets rich in cholesterol and saturated fats (mostly animal) tend to raise serum Total Cholesterol (TC) and Low Density Lipoprotein-Cholesterol (LDL-C). Conversely, a diet low in cholesterol and low in the ratio of saturated-to-polyunsaturated fats lower plasma cholesterol level. Thus, dietary modification remains the first line management of primary hyperlipidemia. Often, intake of SFA is discouraged and that of unsaturated fat (UFA) is encouraged, by doctors. But, probably none of the advices is complete because not all SFAs are hypercholesterolemic and atherogenic. For example, palmitic acid (16:0) and stearic acid (18:0), comprising respectively $90 \%$ and $10 \%$ of total SFAs in palm oil, are neutral and does not elevate blood cholesterol level. ${ }^{4,5,6,7,8}$ Although it has been established that dietary SFAs tend to raise serum cholesterol levels, studies suggest that palm oil, despite a high concentration of SFAs (mainly palmitic acid), is not hypercholesterolemic and is an exception to this general concept. ${ }^{9,10,11}$ In fact, the position of SFA and UFA in triglyceride (TG) backbone of the fat molecule dictates the hypercholesterolemic nature of the fat. ${ }^{12,13,14,15}$ In palm oil $75 \%$ of UFA chains are found at second carbon atom position of the TG backbone molecule $^{16,17}$ and this could explain the nonhypercholesterolemic and non-atherogenic effects of palm oil.

On the other hand excessive consumption of UFAs, in comparison to SFAs, may result in increased susceptibility of body lipids being oxidized (lipid peroxidation) by the highly reactive free oxygen radicals. Lipid peroxidation, in turn, may increase the risk of cell membrane damage as well as accumulation of oxidized LDL (oLDL) particles in the subendothelial layer of blood vessels - a step in the genesis of atherosclerosis.

Now, another deviant fatty acid species, Trans Fatty Acids, have emerged as a new cholesterolemic 'villain' in the field of dietary fats and have been blacklisted by medical and health professionals as contributing to coronary heart disease (CHD). In fact, TFAs have been slowly climbing to the top of infamous list of dietary fats that put us at the risk of heart attack. Though there were some dispute about the significance of TFAs in human nutrition (especially concerning their negative impact on serum lipoprotein profile enhancing atherogenesis), ${ }^{18,19}$ several studies clearly demonstrate that dietary TFAs, relative to their cis isomers, can deleteriously affect serum lipid profile by increasing LDL, decreasing HDL $^{19,20,21,22}$ and raising atherogenic lipoprotein (a) $[\mathrm{Lp}(\mathrm{a})]{ }^{23,24}$ In earlier studies comparison were made considering metabolic effect of exchanging trans 18:1 for cis 18:1 and not SFAs. ${ }^{22}$ Later Judd et al (1994) included a saturated fat comparison. ${ }^{19}$ His study revealed that SFA raised LDL-C similar to that found with 7\% energy from trans, but TFA decreased HDL-C as well. In a Norwegian study butter was used as a saturated fat control for comparison with partially hydrogenated soybean oil (PHSBO) or fish oil (PHFO), where both trans preparations elevated $\mathrm{Lp}(\mathrm{a})$, butter diet group being intermediate between the PHSBO and PHFO. $^{21}$ 
Professor Scott Grundy, in his paper, ${ }^{25}$ commented like this - "there is convincing evidence that trans fatty acids definitely raise LDL-C levels, in a manner similar to that of the cholesterol raising SFA." A Dutch study ${ }^{26}$ concluded, "the effect of trans-fatty acids; it increases the 'bad' LDLcholesterol level and lower the HDL-cholesterol level." Professor Walter C Willett and Dr Albert Ascherio (members of the Harvard University Department of Nutrition and Epidemiology) reviewed the growing science on TFAs and CHD in May 1994 and concluded: "Although the percentage of coronary heart disease deaths in the United States attributable to intake of trans fatty acids is uncertain, even the lower estimates from the effects on blood lipids would suggest that more than 30,000 deaths per year may be due to consumption of partially hydrogenated vegetable fat. Further, the number of attributable cases of non-fatal coronary heart disease will be even larger."27

Sundram et al., in his study, ${ }^{28}$ compared the effects of TFAs with specific SFAs on human lipoprotein profile among the Malaysian population. Finding show an elevation of total plasma cholesterol (TC) with trans-rich diet in comparison to cis 18:1-rich and 16:0-rich diets but similar TC level was found for the trans-rich and 12:0 + 14:0-rich diets. LDLC was slightly higher during trans intake. A marked reduction in HDL-C was noted during trans consumption, which was significantly lower than cis $18: 1$ or $16: 0$ diets. These data affirm a striking difference between the effects of SFAs and TFAs on human lipid profile i.e., TFA depress HDL whereas SFA typically increase HDL along with a rise in LDL.

In addition to the deleterious effects of TFAs on CHD, much interest has been focused on the effects of TFAs on metabolism of essential fatty acids (EFA), all-cis linoleic acid (LA, 18:2n-6) and $\alpha$-linolenic acid (ALA, 18:3n-3), which are vital to fetal growth and development. of TFAs consumption by pregnant women may impair uptake of these EFAs by the fetus. TFAs may also affect the metabolism of EFAs of the fetus resulting in poor development of the fetal organs and tissues. There is increasing evidence that breast milk of lactating mother, who habitually consume food products containing partially hydrogenated fats, contain significant amount of TFAs. Studies revealed that in human breast milk TFA content is inversely related with the concentration of all-cis LA ALA (displacement of these EFAs by TFAs). This FA profile of breast milk of TFA consuming mothers is supposed to affect infant growth and development, adversely. Findings of two European studies showed that there exists significant associations between low birth weights (LBW) and TFA level in blood. ${ }^{29,30,31}$

Another five-center Euramic study suggested of an association between TFAs and increasing breast cancer risk in postmenopausal women. A high trans content was found associated with a $40 \%$ increase in breast cancer risk. ${ }^{32}$

Explanation for the rise in LDL/HDL ratio, with TFA diet, is still not well explored. But this has been attributed to increased cholesteryl ester transfer protein (CETP) activity, i.e., enhanced transfer of cholesteryl ester (CE) from HDL to lower density lipoproteins, including LDL. A 10\% rise in CETP was noted during hydrogenated fat based margarine consumption. ${ }^{20} \mathrm{~A}$ diet, which increases LDL/HDL ratio (especially by increasing the absolute pool of LDL), predisposes CHD risk. This is not only from LDL deposition in arteries but also from a negative effect of elevated LDL/HDL ratio on platelet aggregation and thrombogenesis, an exceedingly deleterious aspect of atherogenesis. ${ }^{32,33}$ Also there is an unhealthy link between TFA intake and the Tumor Necrosis Factor (TNF) system which activates systemic inflammation.

\section{Conclusion}

TFAs are harmful for the heart in the way that they raise LDL level, LDL/HDL ratio (atherogenic effect) as well as Lp (a) and at the same time lower protective HDL to the extent much more than that done by SFAs. TFAs also displace EFAs in human milk affecting infant growth and development. Therefore, foods containing hardened or hydrogenated vegetable and fish oils like margarines, vegetable ghee, confectionary fats etc. prepared from hydrogenated edible oils should be avoided. 


\section{References}

1. Davidson's Principle and Practice of Medicine.

2. Internal Medicine. Hurrison.

3. Harper's Biochemistry

4. Hayes KC, Pronczuk A, Lindsey S and DiersenSchade D (1991). Dietary saturated fatty acids $(12: 0,14: 0,16: 0)$ differ in their impact on plasma cholesterol and lipoproteins in nonhuman primates. American Journal of Clinical Nutrition 53: 491-498.

5. Hayes KC (1993). Specific dietary fat without reducing saturated fatty acids does not significantly lower plasma cholesterol concentrations in normal males. American Journal of Clinical Nutrition 55: 675-681.

6. Hayes KC, Khosla P and Pronczuk A (1995). A rationale for plasma cholesterol modulation by dietary fatty acids: modulating the human response in animals. Journal of Nutritional Biochemistry 6: 188-194.

7. Khosla P and Hayes KC (1992). Comparison between the effects of dietary saturated (16:0), monounsaturated (18:1) and polyunsaturated (18:2) fatty acids on plasma lipoprotein metabolism in Cebus and Rhesus monkeys fed cholesterol-free diets. American Journal of Clinical Nutrition 55: 51-62.

8. Khosla P and Hayes KC (1994). Cholesterolemic effects of the saturated fatty acids of palm oil. Food and Nutrition Bulletin 15: 119-125.

9. Jian Zhang, Wang Ping, Wang Chunrong, Chen Xiao Shou and Ge Keyou (1997). Nonhypercholesterolemic Effects of a Palm Oil Diet in Chinese Adults. The Journal of Nutrition, Vol 127; No 3, March 1997 (Supplement): 509S-513S.

10. Hetzel BS, Charnock JS, Dwyer T and Mclennan PL (1989). Fall in coronary heart disease mortality in USA and Australia due to sudden death: evidence for the role of polyunsaturated fat. Journal of Clinical Epidemiology 42: 855-893.

11. Hornstra G and Sundram K (1991). The influence of dietary palm oil on cardiovascular risk factors: a human study. Abstracts, PORIM International Palm Oil Conference 9-14 September, 1991, Kuala Lumpur, Malaysia. PORIM 1991: 154-155.

12. Innis SM, Quinlan P and Diersan-Schade D (1993). Saturated fatty acid chain length and positional distribution in infant formulas: effects on growth and plasma lipids and ketones in piglets. American Journal of Clinical Nutrition 57: 382-390.

13. Kritchevsky D (1995). Fatty acids, triglyceride structure and lipid metabolism. Journal of Nutritional Biochemistry 6: 172-178.
14. Kritchevsky D (1996). Influence of triglyceride structure on experimental atherosclerosis in rabbits. FASEB Journal 10: A187.

15. Kritchevsky D (1998). Effects of triglyceride structure on lipid metabolism. Nutrition Review 46: 177.

16. Berger KG (1983) Palm Oil. In: Handbook of Tropical Oils. Chan H.T., Jr., eds. Marcel Dekker, New York p448.

17. Padley FB, Gunstone FO and Harwood JL (1986). Occurrence and characterization of oil and fats, in: The Lipid Handbook, Gunstone, F.D., Harwood, J.L. and F.B. Padley eds., Chapman and Halls, New York p49.

18. Willet WC, Stampfer JM, Manson JE, Colditz GA, Speizer FE, Rosner BA, Sampson LA and Hennekens $\mathrm{CH}$ (1993). Intake of trans fatty acids and risk of coronary heart disease among women. Lancet 341: 581-586.

19. Judd JT, Clevidence BA, Muesing RA, Wittes J, Sunkin ME and Podczasy JJ (1994). Dietary trans fatty acids: effects on plasma lipids and lipoproteins of healthy men and women. American Journal of Clinical Nutrition 59: 1245-46.

20. Abbey M and Nestel PJ (1994). Plasma cholesteryl ester transfer protein is increased when transelaidic acid is substituted for cis-oleic acid in the diet. Atherosclerosis 106: 99-107.

21. Almendingen $K$, Jordal $O$, Kierulf $P$, Sandstad $B$ and Pederse $\mathrm{JI}$ (1995). Effects of partially hydrogenated fish oil, partially hydrogenated soybean oil and butter on serum lipoproteins and Lp(a) in men. J. Lipid Res. 36: 1370-1384.

22. Katan MB, Zock PL and Mensink RP (1995). Trans fatty acids and their effects on lipoproteins in humans. Annual Review of Nutrition 15: 473-493.

23. Mensink RP, Zock PL, Katan MB and Hornstra $G$ (1992). Effect of dietary cis and trans fatty acids on serum lipoprotein(a) levels in humans. J. Lipid Res 33: 1493-1501.

24. Nestel PJ, Noakes M, Belling B, McArthur R, Clifton $P$, Janus $E$ and Abbey M (1992). Plasma lipoprotein lipid and $L p(a)$ changes with substitution of elaidic acid for oleic acid in the diet. J. Lipid Res 33: 10291036.

25. Grundy SM (1990). Trans monounsaturated fatty acids and serum cholesterol levels. New England Journal of Medicine 323: 480.

26. Mensink RP and Katan MB (1990). Effects of dietary trans-fatty acids on high-density and lowdensity lipoprotein cholesterol levels in healthy subjects. New England Journal of Medicine 323: 439-445. 
27. Willet WC and Ascherio A (1994). "Trans -fatty Acids: Are the Effects Only Marginal?" American Journal of Public Health 84: 722-724.

28. Sundram K, Ismail A, Hayes KC, Jeyamalar R and Pathmanathan R (1997). Trans (Elaidic) Fatty Acids Adversely Affect the Lipoprotein Profile Relative to Specific Saturated Fatty Acids in Humans. Journal of Nutrition 127:514S-520S.

29. Koletzko B (1992a). Trans fatty acids may impair biosynthesis of long-chain polyunsaturates and growth in man. Acta. Paediatr. Scan 81: 302-306.

30. Koletzko B (1992b). Fats for brain. European Journal of Clinical Nutrition 46: S51-62.
31. Jendryczko A, Gruszynski J, Tomala J and Szpyrka $G$ (1993). Unsaturated fatty acids trans isomers in plasma of pregnant women and birth weight. Ginekol-Pol. 64: 113-116.

32. Kohlmejer L, Simonsen N, Veer $P$ et al (1997). Adipose tissue trans fatty acids and breast cancer in the European community multicenter study on antioxidants, myocardial infarction and breast cancer. Cancer Epidemiology, Biomarkers and Prevention 6: 705-710.

33. Hayes KC and Pronczuk A (1996). Sensitivity to platelet aggregation appears related to the lipoprotein profile and atherosclerosis risk in humans and across species. Comp Biochem. Physiol. 35B: 349-353.
All correspondence to:

Raghib Ahsan Lecturer Department of Physiology Rajshahi Medical College, Rajshahi 